\title{
Alternative approach to electromagnetic field quantization in nonlinear and inhomogeneous media
}

\author{
Lu-Ming Duan and Guang-Can Guo* \\ Physics Department and Nonlinear Science Center, University \\ of Science and Technology of China, Hefei,230026 P.R.China
}

\begin{abstract}
A simple approach is proposed for the quantization of the electromagnetic field in nonlinear and inhomogeneous media. Given the dielectric function and nonlinear susceptibilities, the Hamiltonian of the electromagnetic field is determined completely by this quantization method. From Heisenberg's equations we derive Maxwell's equations for the field operators. When the nonlinearity goes to zero, this quantization method returns to the generalized canonical quantization procedure for linear inhomogeneous media [Phys. Rev. A, 43, 467, 1991]. The explicit Hamiltonians for the second-order and third-order nonlinear quasi-steady-state processes are obtained based on this quantization procedure.
\end{abstract}

PACS numbers:42.50.-p, 42.65.-k, 11.10.Lm

*E-mail:gcguo@sunlx06.nsc.ustc.edu.cn 


\section{Introduction}

Early quantization of the electromagnetic field is performed in empty cavities or in infinite free space [1]. However, with the growth of interest in quantum optical phenomena taking place inside material media, several approaches have been proposed for quantization of the electromagnetic field in nonlinear, inhomogeneous, or dispersive media [2-21]. Early attempts towards quantization of the nonlinear media, while incorporating the known linear theory, did not fully reproduce the nonlinear field equations [4]. An innovative treatment was first proposed by Hillery and Mlodinow who successfully quantized a nonlinear medium by introducing the dual potential [6]. Later, Drummond extended the Hillery-Mlodinow procedure to dispersive media [9]. There are also other approaches in this direction. Glauber and Lewenstein generalized the canonical quantization method by modifying the gauge condition to deal with the inhomogeneous linear media [11]. Abram and Cohen, following the canonical quantization procedure, presented a quantum formulation for light propagation in nonlinear effective media [12]. And recently, Santos and Loudon gave an alternative approach to the quantization of the electromagnetic field in linear one-dimensional dispersive media [19]. Developments towards the absorbing dielectrics also appeared [20,21].

In this paper, we propose a relatively simpler approach to the electromagnetic field quantization in nonlinear and inhomogeneous media. The procedure follows Ref. [11] in using the material independence of the commutation relations for

the fields $\vec{D}$ and $\vec{B}$, pointed out by Born and Infeld [2], as a starting point in the quantization. We extend this to nonlinear media in which $\vec{D}$ and $\vec{B}$ can be expressed as isochronous functionals of the fields $\vec{E}$ and $\vec{H} . \vec{D}$ and $\vec{B}$ are expanded into the mode functions. Furthermore, we explicitly derive Maxwell's 
equations for the field operators from Heisenberg's equations. This procedure is applied to the quantization of the second-order and third-order nonlinear quasisteady-state processes and we obtain the explicit Hamiltonians. Though these Hamiltonians are already in wide use in quantum optics, their derivations are mainly based on the early quantization procedure by Shen $[4,22,23]$ and known by now to be inconsistent with the nonlinear field equations [9]. So here we give a justification of these Hamiltonians.

The arrangement of the paper is as follows. The quantization procedure is proposed in Sec.2. Given dielectric tensor and nonlinear susceptibilities, this quantization procedure completely determines the Hamiltonian of the electromagnetic field, which is expressed by annihilation and creation operators. Then we derive Maxwell's equations from Heisenberg's equations for the field operators. In Sec.3 we show this quantization procedure returns to the generalized canonical quantization method in Ref. [11] when the medium is linear. The explicit Hamiltonians of the second-order and third-order nonlinear quasi-steady-state processes are obtained in Sec. 4 by application of this quantization procedure.

\section{Quantization in the presence of nonlinear me- dia}

We consider the electromagnetic field in nonlinear media, which may be inhomogeneous. The source-free Maxwell equations in matter take the forms [24]

$$
\begin{gathered}
\nabla \cdot \vec{D}=0, \\
\nabla \cdot \vec{B}=0, \\
\frac{1}{c} \frac{\partial \vec{D}}{\partial t}=\nabla \times \vec{H},
\end{gathered}
$$




$$
\frac{1}{c} \frac{\partial \vec{B}}{\partial t}=-\nabla \times \vec{E} .
$$

In nonlinear media, $\vec{D}(t)$ and $\vec{B}(t)$ are complicated nonlinear functionals of $\vec{E}(t)$ and $\vec{H}(t)$. From the Maxwell equations, the energy density $U$ of the electromagnetic field in nonlinear media is determined by

$$
d U(\vec{r}, t)=\vec{E}(\vec{r}, t) \cdot d \vec{D}(\vec{r}, t)+\vec{H}(\vec{r}, t) \cdot d \vec{B}(\vec{r}, t) .
$$

The Hamiltonian (or the energy) is

$$
\widetilde{H}=\int d^{3} \vec{r} U(\vec{r}, t) .
$$

For the electromagnetic field in linear media, the canonical quantization method is generally used. The vector potential $\vec{A}$ is chosen as the general coordinate and the Columb gauge $\nabla \cdot \vec{A}=0$ is often used. $\vec{A}$ and its conjugate momentum can be expanded into a set of transverse complete spatial functions and the expansion coefficients are expressed by annihilation and creation operators. Then substituting the expansions of $\vec{A}$ and its conjugate momentum into the Hamiltonian, one achieves quantization of the electromagnetic field in linear media. However, for the nonlinear media, the canonical quantization becomes much more involved. $\vec{A}$ and $\vec{E}$ were chosen as the canonical variables in the early treatments, which did not incorporate Eq.(1). In fact, no rigorous approach had been proposed for nonlinear media until Hillery and Mlodinow introduced the dual potential and then followed the canonical quantization procedure. Here, inspired by the result in Ref.[1] that the fields $\vec{D}$ and $\vec{B}$ have medium-independent commutation relations in inhomogeneous linear media, we choose the fields $\vec{D}$ and $\vec{B}$, rather than $\vec{E}$ or $\vec{A}$, as the starting point of the electromagnetic field quantization. This choice is also consistent with the results in Ref. [6] [9] and [12], where the field $\vec{D}$ was found to be the canonical momentum. Starting from the mode expansions 
of the fields $\vec{D}$ and $\vec{B}$, we can present a concise formulation of the quantization and a clear derivation of Maxwell's equations for the field operators.

From Equations (1) and (2), the fields $\vec{D}$ and $\vec{B}$ can be expanded into a set of transverse complete spatial functions $\left\{\vec{f}_{\vec{k}_{\mu}}(\vec{r})\right\}$ and $\left\{\nabla \times \vec{f}_{\vec{k}_{\mu}}(\vec{r})\right\}$, respectively,

$$
\begin{gathered}
\vec{D}(\vec{r}, t)=-\sum_{\vec{k} \mu} P_{\vec{k} \mu}(t) \vec{f}_{\vec{k}_{\mu}}^{*}(\vec{r}), \\
\vec{B}(\vec{r}, t)=c \sum_{\vec{k} \mu} Q_{\vec{k}_{\mu}}(t) \nabla \times \vec{f}_{\vec{k}_{\mu}}(\vec{r}) .
\end{gathered}
$$

The expansion functions and coefficients satisfy Hermitian conditions

$$
\begin{aligned}
& \vec{f}_{\vec{k} \mu}^{*}=\vec{f}_{-\vec{k} \mu}, \\
& Q_{\vec{k} \mu}^{+}=Q_{-\vec{k} \mu}, \\
& P_{\vec{k} \mu}^{+}=P_{-\vec{k} \mu} .
\end{aligned}
$$

In addition, the functions $\vec{f}_{\vec{k} \mu}(\vec{r})$ satisfy transversality, orthonormality and completeness conditions

$$
\begin{gathered}
\nabla \cdot \vec{f}_{\vec{k} \mu}=0, \\
\int d^{3} \vec{r}^{f_{\vec{k}_{\mu i}}^{*}}(\vec{r}) \vec{f}_{\vec{k}^{\prime} \mu^{\prime} j}(\vec{r})=\delta_{\vec{k} \vec{k}^{\prime}} \delta_{\mu \mu^{\prime}} \delta_{i j}, \\
\sum_{\vec{k} \mu} \vec{f}_{\vec{k}_{\mu i}}^{*}(\vec{r}) \vec{f}_{\vec{k}_{\mu j}}\left(\vec{r}^{\prime}\right)=\delta_{i j}^{T}\left(\vec{r}-\vec{r}^{\prime}\right),
\end{gathered}
$$

where the transverse $\delta$-function $\delta_{T}$ is defined as

$$
\delta_{i j}^{T}(\vec{r})=\frac{1}{(2 \pi)^{3}} \int d^{3} \vec{k}\left(\delta_{i j}-\frac{k_{i} k_{j}}{|\vec{k}|^{2}}\right) e^{i \vec{k} \cdot \vec{r}} .
$$

The transversality condition (12) makes the completeness equation of $\vec{f}_{\vec{k}}{ }_{\mu}(\vec{r})$ take the form of (14). In free space, the plane wave is chosen as the expansion 
function

$$
\vec{f}_{\vec{k} \mu}(\vec{r})=\frac{1}{(2 \pi)^{\frac{3}{2}}} \vec{e}_{\vec{k} \mu} e^{i \vec{k} \cdot \vec{r}},
$$

where the unit vectors $\vec{e}_{\vec{k}_{\mu}}(\mu=1,2)$ satisfy

$$
\vec{k} \cdot \vec{e}_{\vec{k} \mu}=0
$$

The expansions (7) and (8) have the same forms as those in linear media. We further suppose that the expansion coefficient operators satisfy the same commutation relations. So

$$
\left[Q_{\vec{k} \mu}(t), P_{\vec{k}^{\prime} \mu^{\prime}}(t)\right]=i \hbar \delta_{\vec{k} \vec{k}^{\prime}} \delta_{\mu \mu^{\prime}}
$$

The fields $\vec{E}(\vec{r}, t)$ and $\vec{H}(\vec{r}, t)$ can be expressed by $\vec{D}(\vec{r}, t)$ and $\vec{B}(\vec{r}, t)$ from the nonlinear functional relations between them. From (5) and (6) the Hamiltonian of the electromagnetic field becomes a nonlinear functional of $\vec{D}(\vec{r}, t)$ and $\vec{B}(\vec{r}, t)$. After substituting the expansions (7) and (8) into it, we get the Hamiltonian, which is expressed by annihilation and creation operators. Given the functional relations between $\vec{E}(\vec{r}, t), \vec{H}(\vec{r}, t)$ and $\vec{D}(\vec{r}, t), \vec{B}(\vec{r}, t)$, the Hamiltonian form is completely determined by this quantization procedure.

Now we show reasonableness of the quantization method. Comparing (7),(8) and (18) with the corresponding equations in Ref.[1], we know that when the nonlinearity goes to zero the above procedure returns to the generalized canonical quantization method. Furthermore, this quantization gives the correct Maxwell equations. In the following we derive Maxwell's equations for the field operators from Heisenberg's equations.

From the transversality of the expansion functions $\vec{f}_{\vec{k} \mu}$, the first two Maxwell equations (1) and (2) are obviously satisfied. Equations (7) (8) and (14) (18) give 
the commutator of the field operators $\vec{D}$ and $\vec{B}$

$$
\left[D_{i}(\vec{r}, t), B_{j}\left(\vec{r}^{\prime}, t\right)\right]=i \hbar c(\nabla \times \delta)_{i j}\left(\vec{r}-\vec{r}^{\prime}\right) .
$$

In the derivation, the following relations are used.

$$
\begin{gathered}
\left(\nabla \times \delta^{T}\right)_{i j}(\vec{r})=\sum_{m n} \varepsilon_{i m n} \partial_{m} \frac{1}{(2 \pi)^{3}} \int d^{3} \vec{k}\left(\delta_{n j}-\frac{k_{n} k_{j}}{|\vec{k}|^{2}}\right) e^{i \vec{k} \cdot \vec{r}} \\
=\sum_{m n} \varepsilon_{i m n} \partial_{m} \frac{1}{(2 \pi)^{3}} \int d^{3} \vec{k} \delta_{n j} e^{i \vec{k} \cdot \vec{r}} \\
=(\nabla \times \delta)_{i j}(\vec{r})
\end{gathered}
$$

and

$$
\begin{gathered}
\left(\nabla^{\prime} \times \delta\right)_{j i}\left(\vec{r}-\vec{r}^{\prime}\right)=-(\nabla \times \delta)_{j i}\left(\vec{r}-\vec{r}^{\prime}\right) \\
=-\sum_{m n} \varepsilon_{j m n} \partial_{m} \delta_{n i}\left(\vec{r}-\vec{r}^{\prime}\right) \\
=-\sum_{m} \varepsilon_{j m i} \partial_{m} \delta\left(\vec{r}-\vec{r}^{\prime}\right) \\
=\sum_{m} \varepsilon_{i m j} \partial_{m} \delta\left(\vec{r}-\vec{r}^{\prime}\right) \\
=(\nabla \times \delta)_{i j}\left(\vec{r}-\vec{r}^{\prime}\right) .
\end{gathered}
$$

The commutator (19) has been given in Ref. [12] in one-dimensional form. Here we extend it to the general case. From the commutator (19), the commutator between $\vec{D}$ or $\vec{B}$ and an arbitrary functional $F(\vec{D}, \vec{B})$ of $\vec{D}$ and $\vec{B}$, which may be nonlinear, can be expressed by functional derivation as follows:

$$
\begin{gathered}
{\left[D_{i}(\vec{r}, t), F\right]=i \hbar c \sum_{m n} \varepsilon_{i m n} \partial_{m} \frac{\delta}{\delta B_{n}(\vec{r}, t)} F} \\
{\left[B_{i}(\vec{r}, t), F\right]=-i \hbar c \sum_{m n} \varepsilon_{i m n} \partial_{m} \frac{\delta}{\delta D_{n}(\vec{r}, t)} F .}
\end{gathered}
$$

From these two equations and (5),(6), the Heisenberg equations of the field op- 
erators $\vec{D}$ and $\vec{B}$ take the forms

$$
\begin{gathered}
\frac{1}{c} \frac{\partial D_{i}}{\partial t}=\frac{1}{i \hbar c}\left[D_{i}(\vec{r}, t), \widetilde{H}\right] \\
=\sum_{m n} \varepsilon_{i m n} \partial_{m} \frac{\delta}{\delta B_{n}(\vec{r}, t)} \widetilde{H} \\
=\sum_{m n} \varepsilon_{i m n} \partial_{m} \frac{\partial U(\vec{r}, t)}{\partial B_{n}(\vec{r}, t)} \\
=\sum_{m n} \sum_{j} \varepsilon_{i m n} \partial_{m} H_{j} \frac{\partial B_{j}}{\partial B_{n}} \\
=(\nabla \times \vec{H})_{i} .
\end{gathered}
$$

Similarly

$$
\frac{1}{c} \frac{\partial B_{i}}{\partial t}=-(\nabla \times \vec{E})_{i}
$$

So we have clearly derived Maxwell's equations for the field operators from Heisenberg's equations. The isochronous commutators (22),(23) play an essential role in the derivation. The derivation holds for linear or nonlinear media. However, for dispersive media, nonlocal relations in time between the Hamiltonian $\widetilde{H}$ and the fields $\vec{D}, \vec{B}$ arise and the isochronous commutators (22),(23) cannot be applied in this case. Here we meet the long standing difficulty in quantum optics in quantizing nonlinear and dispersive dielectrics.

\section{Quantization of the electromagnetic field in linear inhomogeneous media}

In this section we use the above method to quantize the electromagnetic field in linear inhomogeneous media. The medium is characterized by

$$
\begin{gathered}
D_{i}(\vec{r}, t)=\sum_{j} \varepsilon_{i j}(\vec{r}) E_{j}(\vec{r}, t), \\
\vec{B}(\vec{r}, t)=\vec{H}(\vec{r}, t) .
\end{gathered}
$$


The Hamiltonian (or the energy) is

$$
\widetilde{H}=\frac{1}{2} \int d^{3} \vec{r}\left(\vec{B}^{2}+\sum_{i j} \varepsilon_{i j}^{-1} D_{i} D_{j}\right) .
$$

In free space the expansion function $\vec{f}_{\vec{k} \mu}$ is expressed by Eq.(16). Substituting the expansions (7) and (8) into Eq.(28) and noting

$$
\begin{gathered}
Q_{\vec{k} \mu}(t)=\left(\frac{\hbar}{2 \omega_{\vec{k} \mu}}\right)^{\frac{1}{2}}\left(a_{\vec{k} \mu}+a_{-\vec{k} \mu}^{+}\right), \\
P_{\vec{k} \mu}(t)=i\left(\frac{\hbar \omega_{\vec{k} \mu}}{2}\right)^{\frac{1}{2}}\left(a_{\vec{k} \mu}^{+}-a_{-\vec{k} \mu}\right),
\end{gathered}
$$

where $\omega_{\vec{k} \mu}=|\vec{k}| c$, we get the Hamiltonian expressed by annihilation and creation operators.

$$
\begin{gathered}
\widetilde{H}=\sum_{\vec{k} \mu^{\prime}} \hbar \omega_{\vec{k} \mu} a_{\vec{k} \mu}^{+} a_{\vec{k} \mu}+\frac{\hbar}{4} \sum_{\vec{k} \vec{k}^{\prime} \mu^{\prime}} \sqrt{\omega_{\vec{k} \mu} \omega_{\vec{k}^{\prime} \mu^{\prime}}} \\
\times\left[V_{\mu \mu^{\prime}}^{*}\left(\vec{k}, \vec{k}^{\prime}\right) a_{\vec{k} \mu}^{+} a_{\vec{k}^{\prime} \mu^{\prime}}^{+}-V_{\mu \mu^{\prime}}^{*}(\vec{k},-\vec{k}) a_{\vec{k} \mu}^{+} a_{\vec{k}^{\prime} \mu^{\prime}}+\text { h.c. }\right],
\end{gathered}
$$

where $V_{\mu \mu^{\prime}}$ is defined by

$$
V_{\mu \mu^{\prime}}\left(\vec{k}, \vec{k}^{\prime}\right)=\frac{1}{(2 \pi)^{3}} \int d^{3} \vec{r}^{e_{\vec{k} \mu}} \overrightarrow{\vec{k}} \cdot\left(1-\epsilon^{-1}\right) \cdot \vec{e}_{\vec{k}^{\prime} \mu^{\prime}} e^{i\left(\vec{k}+\vec{k}^{\prime}\right) \cdot \vec{r}}
$$

If the second-order tensor $\varepsilon^{-1}$ is a scalar, $\vec{e}_{\vec{k} \mu} \cdot \vec{e}_{\vec{k}^{\prime} \mu^{\prime}}$ in Eq.(32) can be put out of the integration. This case had been discussed in detail in Ref.[11] by the generalized canonical quantization method. Here we get the same results.

\section{Quantization of the quasi-steady-state optical field in nonlinear media}

In this section we consider quantization of the quasi-steady-state optical field in nonlinear media. The second-order or third-order nonlinear process is most important $[25,23]$. First we apply the quantization method to the parametric 
process. The optical field is composed of three quasi-monochromatic fields with central frequency $\omega_{1}, \omega_{2}, \omega_{3}$, respectively, and $\omega_{3}=\omega_{1}+\omega_{2}$, i.e.,

$$
\vec{E}(t)=\sum_{i=1}^{3} \vec{E}^{(i)}(t) e^{-i \omega_{i} t}+\text { h.c. }
$$

where $\vec{E}^{(i)}(t)$ is slowly varying amplitude. Under the quasi-steady-state approximation, the term $\vec{E}^{(i)}(t) e^{-i \omega_{i} t}$ in Eq.(33) can be viewed as a monochromatic field with frequency $\omega_{i}$, i.e., the dispersion of the optical field in the medium is negligible. Suppose the refractive index is independent of orientation of space. Then the field $\vec{D}^{(i)}(t)$ can be expressed by $\vec{E}^{(i)}(t)$. For example,

$$
\vec{D}^{(3)}(t)=n^{2}\left(\omega_{3}\right) \vec{E}^{(3)}(t)+\chi^{(2)}\left(\omega_{3}=\omega_{1}+\omega_{2}\right): \vec{E}^{(1)}(t) \vec{E}^{(2)}(t),
$$

where $n^{2}\left(\omega_{3}\right)$ is introduced phenomenologically to show the component of the optical field $\vec{E}^{(3)}(t)$ has been viewed as a monochromatic field with frequency $\omega_{3} . \vec{D}^{(2)}(t)$ and $\vec{D}^{(1)}(t)$ have similar expressions. From these equations, $\vec{E}^{(i)}(t)$ $(i=1,2,3)$ may be expressed by $\vec{D}^{(i)}(t)$ as

$$
\vec{E}^{(3)}(t)=\frac{\vec{D}^{(3)}(t)}{n^{2}\left(\omega_{3}\right)}-\gamma^{(2)}\left(\omega_{3}=\omega_{1}+\omega_{2}\right): \vec{D}^{(1)}(t) \vec{D}^{(2)}(t) \text {, et.al., }
$$

where

$$
\gamma^{(2)}\left(\omega_{3}=\omega_{1}+\omega_{2}\right)=\frac{1}{n^{2}\left(\omega_{1}\right) n^{2}\left(\omega_{2}\right) n^{2}\left(\omega_{3}\right)} \chi^{(2)}\left(\omega_{3}=\omega_{1}+\omega_{2}\right)
$$

In the derivation of Eq.(35) the approximation that the nonlinear terms are much smaller than the linear terms is used. Substituting (35) into (5) and (6) and making the rotating wave approximation, we get the Hamiltonian of the electromagnetic field, which is expressed by $\vec{D}$ and $\vec{B}$

$$
\begin{aligned}
\widetilde{H} & =\frac{1}{2} \int d^{3} \vec{r}\left\{\sum_{i=1}^{3}\left[\frac{\left(\vec{D}^{(i)}(t) e^{-i \omega_{i} t}+h . c .\right)^{2}}{n^{2}\left(\omega_{i}\right)}+\left(\vec{B}^{(i)}(t) e^{-i \omega_{i} t}+h . c .\right)^{2}\right]\right. \\
& \left.-2\left[\gamma^{(2)}\left(\omega_{3}=\omega_{1}+\omega_{2}\right): \vec{D}^{(3)+(t)} \vec{D}^{(2)}(t) \vec{D}^{(1)}(t)+\text { h.c. }\right]\right\}
\end{aligned}
$$


In the derivation, the holo-exchange symmetry of the tensor $\chi^{(2)}$ has been used. In the expansions $(7)$ and (8) of the fields $\vec{D}, \vec{B}$, only the terms with the subscripts $|\vec{k}|=k_{i}$, where $k_{i}=\frac{n\left(\omega_{i}\right) \omega_{i}}{c},(i=1,2,3)$, make contributions to the interaction. So the expansions can be simplified to

$$
\begin{gathered}
\vec{D}^{(i)}(t) e^{-i \omega_{i} t}=i \sqrt{\frac{\hbar \omega_{i} n^{2}\left(\omega_{i}\right)}{2}} \sum_{|\vec{k}|=k_{i}} \sum_{\mu} \vec{f}_{\vec{k} \mu} a_{\vec{k} \mu}(t), \\
\vec{B}^{(i)}(t) e^{-i \omega_{i} t}=c \sqrt{\frac{\hbar}{2 \omega_{i} n^{2}\left(\omega_{i}\right)}} \sum_{|\vec{k}|=k_{i}} \sum_{\mu} \nabla \times \vec{f}_{\vec{k} \mu} a_{\vec{k} \mu}(t) .
\end{gathered}
$$

The function $\vec{f}_{\vec{k} \mu}$ can be decomposed as $\vec{f}_{\vec{k} \mu}=f_{\vec{k}}(\vec{r}) \cdot \vec{e}_{\vec{k} \mu}$, where $f_{\vec{k}}(\vec{r})$ satisfies the eigen-equation

$$
\nabla^{2} f_{\vec{k}}(\vec{r})=-|\vec{k}|^{2} f_{\vec{k}}(\vec{r})
$$

Substituting (38) and (39) into Eq.(37), we get the Hamiltonian expressed by annihilation and creation operators

$$
\begin{gathered}
\widetilde{H}=\sum_{\vec{k}_{1}, \vec{k}_{2}, \vec{k}_{3} \mu_{1}, \mu_{2}, \mu_{3}}\left[\sum_{i=1}^{3} \hbar \omega_{i} a_{\vec{k}_{i} \mu_{i}}^{+} a_{\vec{k}_{i} \mu_{i}}\right. \\
\left.+\left(\alpha_{\vec{k}_{1} \mu_{1} \vec{k}_{2} \mu_{2} \vec{k}_{3} \mu_{3}} \beta_{\vec{k}_{1} \vec{k}_{2} \vec{k}_{3}} a_{\vec{k}_{3} \mu_{3}}^{+} a_{\vec{k}_{2} \mu_{2}} a_{\vec{k}_{1} \mu_{1}}+\text { h.c. }\right)\right],
\end{gathered}
$$

where the constant $\beta$ (called phase-matching factor) is defined as

$$
\beta_{\vec{k}_{1} \vec{k}_{2} \vec{k}_{3}}=\sqrt{V} \int f_{\vec{k}_{3}}^{*} f_{\vec{k}_{2}} f_{\vec{k}_{1}} d^{3} \vec{r}
$$

and $\mathrm{V}$ is volume of the nonlinear media. The constant $\alpha$ is

$$
\begin{aligned}
& \alpha_{\vec{k}_{1} \mu_{1} \vec{k}_{2} \mu_{2} \vec{k}_{3} \mu_{3}}=-i \sqrt{\frac{\hbar^{3} \omega_{1} \omega_{2} \omega_{3}}{8 V n^{2}\left(\omega_{1}\right) n^{2}\left(\omega_{2}\right) n^{2}\left(\omega_{3}\right)}} \\
& x^{(2)}\left(\omega_{3}=\omega_{1}+\omega_{2}\right) \vdots \vec{e}_{\vec{k}_{3} \mu_{3}} \vec{e}_{\vec{k}_{2} \mu_{2}} \vec{e}_{\vec{k}_{1} \mu_{1}}
\end{aligned}
$$


and it is determined by the polarization matching condition of the optical field. When $\vec{k}_{1}, \vec{k}_{2}, \vec{k}_{3}$ are collinear, the Hamiltonian (41) can be simplified. Suppose the polarizations of the optical fields are given (indicated by $\mu_{1}^{\prime}, \mu_{2}^{\prime}, \mu_{3}^{\prime}$ respectively) and $\vec{k}_{i}=k_{i} \vec{e}_{z}(i=1,2,3)$, where $\vec{e}_{z}$ is the unit vector of z-axis. The expansion function is approximately a plane wave, i.e.,

$$
f_{k_{i}}(\vec{r})=S_{k_{i}}(x, y) \frac{e^{-i k_{i} z}}{\sqrt{L}}
$$

where $\mathrm{L}$ is the interaction length. Let $a_{i}$ represent the operator $a_{k_{i} \mu_{i}^{\prime}}$ and

$$
\alpha=\alpha_{k_{1} \mu_{1}^{\prime} k_{2} \mu_{2}^{\prime} k_{3} \mu_{3}^{\prime}} \sqrt{\frac{V}{L}} \int S_{k_{3}}^{*} S_{k_{2}} S_{k_{1}} d x d y
$$

then the Hamiltonian (41) is simplified to

$$
\widetilde{H}=\sum_{i=1}^{3} \hbar \omega_{i} a_{i}^{+} a_{i}+\left(\alpha \cdot \frac{e^{i \Delta k L}-1}{i \Delta k L} a_{3}^{+} a_{2} a_{1}+h . c .\right)
$$

where the phase mismatch $\Delta k=k_{1}+k_{2}-k_{3}$. Eq.(46) is often used to analyze quantum properties of the parametric process [26-28]. Here we give its exact derivation and determine the expression of the parameter $\alpha$.

The optical field in the third-order nonlinear medium can be quantized in a similar way. For example, the Hamiltonian of the nondegenerate four-wavemixing process [23] with $\omega_{3}+\omega_{4}=\omega_{1}+\omega_{2}$ is

$$
\begin{aligned}
& \widetilde{H}=\sum_{\vec{k}_{1}, \vec{k}_{2}, \vec{k}_{3}, \vec{k}_{4}}^{\left|\vec{k}_{i}\right|=k_{i}, \mu_{2}, \mu_{3}, \mu_{4}} \sum_{i=1}^{4} \hbar \omega_{i} a_{\vec{k}_{i} \mu_{i}}^{+} a_{\vec{k}_{i} \mu_{i}}
\end{aligned}
$$

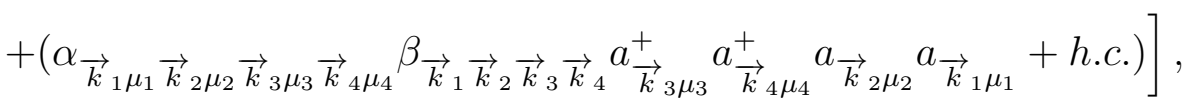

where

$$
\beta_{\vec{k}_{1} \vec{k}_{2} \vec{k}_{3} \vec{k}_{4}}=V \int f_{\vec{k}_{4}}^{*} f_{\vec{k}_{3}}^{*} f_{\vec{k}_{2}} f_{\vec{k}_{1}} d^{3} \vec{r}
$$


and

$$
\begin{gathered}
\alpha_{\vec{k}_{1} \mu_{1} \vec{k}_{2} \mu_{2} \vec{k}_{3} \mu_{3} \vec{k}_{4} \mu_{4}}=-i \sqrt{\frac{\hbar^{4} \omega_{1} \omega_{2} \omega_{3} \omega_{4}}{16 V^{2} n^{2}\left(\omega_{1}\right) n^{2}\left(\omega_{2}\right) n^{2}\left(\omega_{3}\right) n^{2}\left(\omega_{4}\right)}} \\
x^{(3)}\left(\omega_{4}=-\omega_{3}+\omega_{2}+\omega_{1}\right) \vdots \vec{e}_{\vec{k}_{4} \mu_{4}}^{*} \vec{e}_{\vec{k}_{3} \mu_{3}}^{*} \vec{e}_{\vec{k}_{2} \mu_{2}} \vec{e}_{\vec{k}_{1} \mu_{1}}
\end{gathered}
$$

The Hamiltonians (41) and (47) make the foundation for analyzing quantum properties of the parametric or four-wave-mixing process.

\section{Acknowledgment}

This project was supported by the National Nature Foundation of China. 


\section{References}

[1] W.H.Louisell, Quantum Statistical Properties of Radiation, Tohn Wiely (1973).

[2] M.Born and L.Infeld, Proc. R. Soc. London Sev. A 147, 522 (1934); 150,141 $(1935)$

[3] J.M.Jauch and K.M.Watson, Phys. Rev. 74, 950 (1948)

[4] Y.R.Shen, Phys. Rev. 155, 921 (1967).

[5] G.Nienhuis and C.Th.J.Alkemade, Physica 81c, 181 (1976).

[6] M.Hillery and L.D.Mlodinow, Phys. Rev. A 30, 1860 (1984).

[7] I.Abram, Phys. Rev. A 35, 4661 (1987).

[8] L.Knöll, W.Vogel, and D.G.Welsch, Phys. Rev. A 36, 3803 (1988); Prog. Quantum Electron. 16, 135 (1992).

[9] P.D.Drummond, Phys. Rev. A 42, 6845 (1990).

[10] K.J.Blow, R.Loudon, S.J.D.Phoenix, and T.J.Shepherd, Phys. Rev. A 42, 4102 (1990).

[11] R.J.Glauber and M.Lewenstein, Phys. Rev. A 43, 467 (1991).

[12] I.Abram and E.Cohen, Phys. Rev. A 44, 500 (1991).

[13] B.Huttner, J.J.Baumberg, and S.M.Barnett, Europhys. Lett. 16, 177 (1991).

[14] B.Huttner, S.M.Barnett, Europhys. Lett. 18, 487 (1992); Phys. Rev. A 16, 4306 (1992). 
[15] H.Khosravi and R.Loudon, Proc. R. Soc. London, Ser. A 433, 337 (1991); 436, 373 (1992).

[16] S.-T.Ho.and P.Kumar, J. Opt. Soc. Am. B 10, 1620 (1993).

[17] D.D.Marcenac and J.E.Carroll, IEE Proc. J. 140, 157(1993).

[18] A.N.Kireev and M.A.Dupertuis, Mod. Phys. Lett. 7, 1633 (1993).

[19] D.J.Santos and R.Loudon, Phys. Rev. A 52, 1538 (1995).

[20] T.Gruner and D.-G.Welsch, Phys. Rev. A 51, 3246 (1995).

[21] R.Matloob and R.Loudon, Phys. Rev. A 53, 4567 (1996).

[22] M.H.Rubin, D.N.Klyshko, Y.H.Shih and A.V.Sergienko, Phys. Rev. A 50, $5122(1994)$.

[23] Y.R.Shen, the Principles of Nonlinear Optics, John Wiley, (1984).

[24] D.Jackson, Classical Electrodynamics, John Wiley, New York, (1962).

[25] N.Bloembergen, Nonlinear Optics, Benjamin, New York, (1965).

[26] R.J.Thompson, G.Rempe, and H.J.Kimble, Phys. Rev. Lett. 68, 1132 (1992).

[27] R.E.Slusher, Opt. Photon News 4, 8 (1993).

[28] C.J.Mertens, J.M.Hasty, H.H.Roark, D.Nowakawski, and T.A.B.Kennedy, Phys. Rev. A 52, 744 (1995). 